Rhabdomyosarcoma (RMS) is a malignant form of neoplasm that originates from skeletal muscle. RMSs can exist anywhere in the human body but are more commonly detected in the neck region and extremities. The alveolar type is one of the subtypes of RMS that has a poor prognosis. Because the clinical manifestation of a tumour can be a painless mass, symptoms might be non-contributary to the diagnosis. Herein, a four-month-old girl was admitted to the emergency department with complaints of respiratory distress without a runny nose, cough, and fever. Recurrent effusions subsided with subsequent tube thoracostomy. Video-assisted thoracoscopic surgery (VATS) was performed to determine the aetiology of the recurrent effusion. The Tru-Cut biopsy obtained during VATS resulted in the diagnosis of alveolar rhabdomyosarcoma. Pleural effusion decreased, and the tube drainage was stopped rapidly after first vincristine, actinomycin-D, and cyclophosphamide chemotherapy cycle. Persistent and recurrent pleura effusions should alert physicians to rule out unusual diagnoses like that of our case.

Key words: congenital alveolar rhabdomyosarcoma, childhood, pleural effusion.

Contemp Oncol (Pozn) 2020; 24 (2): 132-135 DOI: https://doi.org/10.5114/wo.2020.97639

\section{Persistent pleural effusion in an infant with an unusual diagnosis: congenital alveolar rhabdomyosarcoma}

\author{
Can Yilmaz Yozgat ${ }^{1}$, Osman Yesilbas ${ }^{2}$, Yilmaz Yozgat ${ }^{3}$, Osman Cemil Akdemir ${ }^{4}$, \\ Ismail Yurtsever ${ }^{5}$, Nur Tekin ${ }^{6}$, Damat Baghishov ${ }^{6}$, Nigar Bayramova ${ }^{6}$, \\ Sahande Elagoz ${ }^{7}$, Fatma Betul Cakir ${ }^{8}$ \\ ${ }^{1}$ Faculty of Medicine, Bezmialem Vakif University, Istanbul, Turkey \\ ${ }^{2}$ Department of Paediatric Critical Care Medicine, Bezmialem Vakif University, Istanbul, \\ Turkey \\ ${ }^{3}$ Department of Paediatric Cardiology, Bezmialem Vakif University, Istanbul, Turkey \\ ${ }^{4}$ Department of Thoracic Surgery, Bezmialem Vakif University, Istanbul, Turkey \\ Department of Radiology, Bezmialem Vakif University, Istanbul, Turkey \\ ${ }^{6}$ Department of Paediatrics, Bezmialem Vakif University, Istanbul, Turkey \\ ${ }^{7}$ Department of Pathology, Bezmialem Vakif University, Istanbul, Turkey \\ ${ }^{8}$ Department of Paediatric Haematology and Oncology, Bezmialem Vakif University, \\ Istanbul, Turkey
}

\section{Introduction}

The most frequent causes of pleural effusions are infections in childhood. Malignant thoracic neoplasms, particularly in infancy, are rare in the differential diagnosis of pleural effusion. Although rhabdomyosarcoma (RMS) is an uncommon childhood malignancy, it is the most frequently encountered soft tissue sarcoma found in infants and children. RMSs can exist anywhere in the human body, including head and neck, extremities, genitourinary tract, and trunk as the most common primary sites [1-3]. The alveolar type of RMS is one of the subtypes of RMS that has a poor prognosis. It occurs at a rate of $20 \%$ in all of RMS cases. The tumour pathophysiology is extremely close to the lungs; therefore, the tumour is also known as alveolar rhabdomyosarcoma (ARMS). Congenital ARMS, defined as disease present at birth, is a rare subtype of RMS [4,5]. We present a case of congenital ARMS, in which the patient rapidly improved just after the first chemotherapy cycle.

\section{Case report}

A four-month-old girl was admitted to the emergency department (ED) with complaints of respiratory distress without a runny nose and cough. In her history, she had a rash while having a bath one day earlier. In her physical examination, there was tachypnoea (40 breaths per minute) and a slight tachycardia (120 beats per minute) with no fever. Respiratory sounds in the right lung were diminished. Pleural effusion on the right hemithorax and pneumothorax on the left side was detected in the patient's chest X-ray (Fig. 1). Complete blood count and routine biochemical tests were within normal limits. The levels of C-reactive protein (CRP) and procalcitonin were normal, with no signs of infection.

A full panel viral swab culture was taken from the patient in order to rule out viral infection. The result of the swab culture was positive in terms of rhinovirus. A thorax tube was inserted for the pneumothorax in the left hemithorax, and thoracentesis was performed on the right side. Puncture fluid with $100 \mathrm{cc}$ volume was transudate in character. The patient was transferred to the paediatric intensive care unit (PICU) for differential diagnosis and intensive treatment. On the second day of PICU admission, the effusion re- 
developed in the right hemithorax of the patient. Therefore, a thorax tube was inserted into the right hemithorax. The tube on the left side was removed on the third day of PICU admission. Despite draining approximately $100 \mathrm{cc}$ of fluid from the right hemithorax, effusion persisted for the following two days, and video-assisted thoracoscopic surgery (VATS) was performed to determine the aetiology of the persistent effusion. Pleural thickening with multiple nodules on the diaphragm was detected during VATS operation, and a Tru-Cut biopsy was obtained from the nodules. PAX3/FOXO1 fusion gene analysis confirmed the alveolar subtype and the poor prognosis of the patient. For staging purposes, the patient underwent thoracic and abdominal computed tomography (CT). A $55 \times 32 \times$ $35 \mathrm{~mm}$ heterogeneous semisolid mass, which compresses the liver in the subdiaphragmatic area, was detected (Fig. 2). There was also nodular thickening with $12 \mathrm{~mm}$ size on the right hemithorax pleural face with large pathological lymphadenopathies in the mediastinum (Fig. 3) and around the portal vein of the abdomen. A bony metastasis was detected at the $L 5$ vertebra. Bone marrow aspiration was within normal limits. The pathology resulted in alveolar type RMS consistent with myogenin and desmin positivity (Fig. 4). Thus, the patient was classified as group IV according to the Intergroup Rhabdomyosarcoma Study Group (IRSG) classification, and as stage IV according to TNM pretreatment staging classification [6]. A vincristine, actinomycin-D, and cyclophosphamide (VAC) chemotherapy regimen was started. Because the patient had a plevral effusion during the VATS procedure, a third thorax tube had to be inserted in the right hemithorax. The patient responded to the chemotherapy, and during 10 days of PICU admission the effusion subsided gradually, and all the tubes were taken out. The patient was discharged on the $12^{\text {th }}$ day of PICU admission, and she is still under chemotherapy and followed by the paediatric haematology and oncology division. After four cycles of chemotherapy, the majority of the primary tumour and the metastasis of the pleura, abdominal LAP, and the bony metastasis at the vertebra regressed. At the tumour board, since a very morbid surgery of right hemi pleurectomy was recommended as the local therapy, we decided to continue with chemotherapy only. Radiotherapy (RT) was not planned due to the very young age of the patient.

\section{Discussion}

RMS usually occurs in the first two decades of life. In $5-10 \%$ of cases, it is diagnosed in children aged less than one year, and congenital RMS occurs in $<1 \%$ of all patients with RMS [7, 8]. Only a few cases (less than 20) have been reported in the literature regarding the alveolar subtype of congenital RMS. Various reports indicate that, when the diagnosis occurs in the neonatal period, the prognosis is worse than in older children [9-11]. The most frequent primary site for ARMS is the extremities, whereas congenital ARMS is a highly malignant tumour, often occurring as a disseminated disease $[12,13]$. Likewise, in the literature, our case has poor prognostic characteristics: the alveolar type, large size $(>5 \mathrm{~cm})$ and unfavourable localisation of the primary

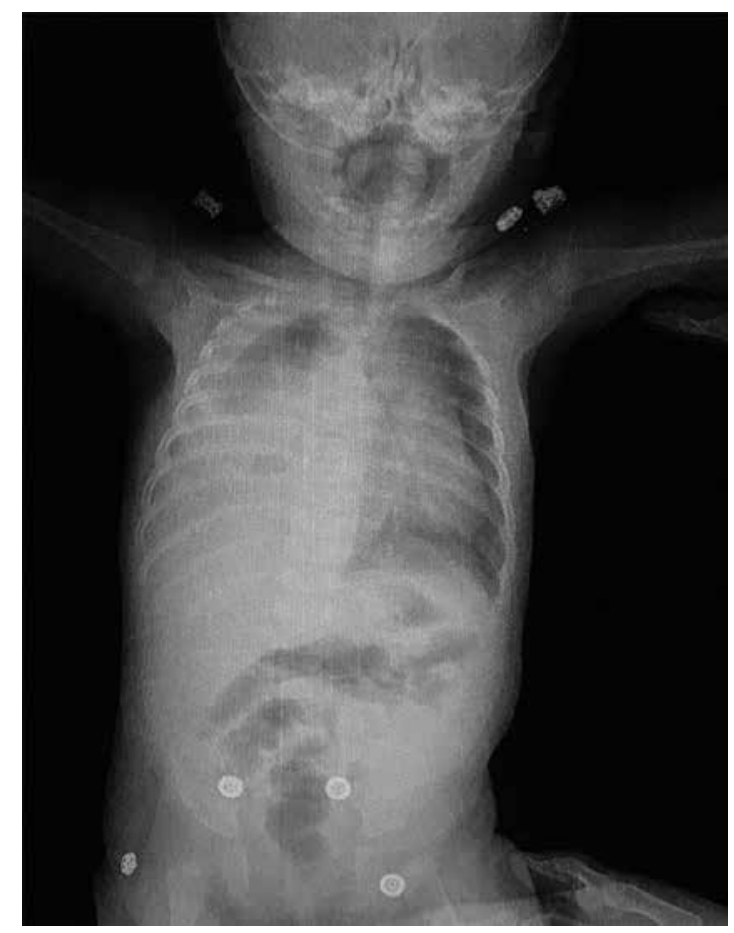

Fig. 1. Chest $\mathrm{X}$-ray showing pleural effusion on the right hemithorax and pneumothorax on the left side.

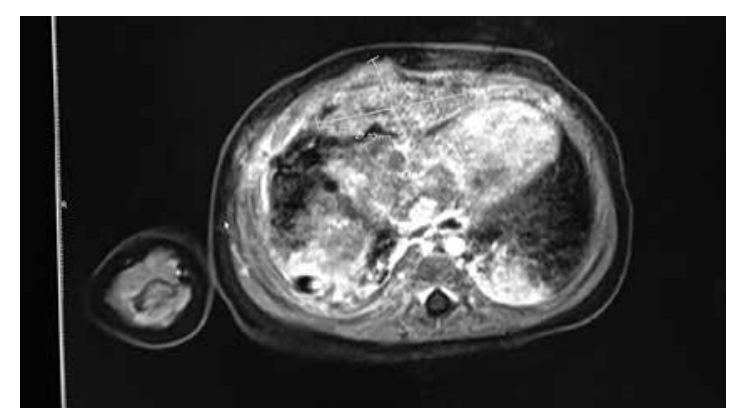

Fig. 2. Contrast-enhanced thorax CT showing a tumour $55 \mathrm{~mm} \times 32$ $\mathrm{mm} \times 35 \mathrm{~mm}$ heterogeneous semisolid mass compressing the liver in the subdiaphragmatic area.

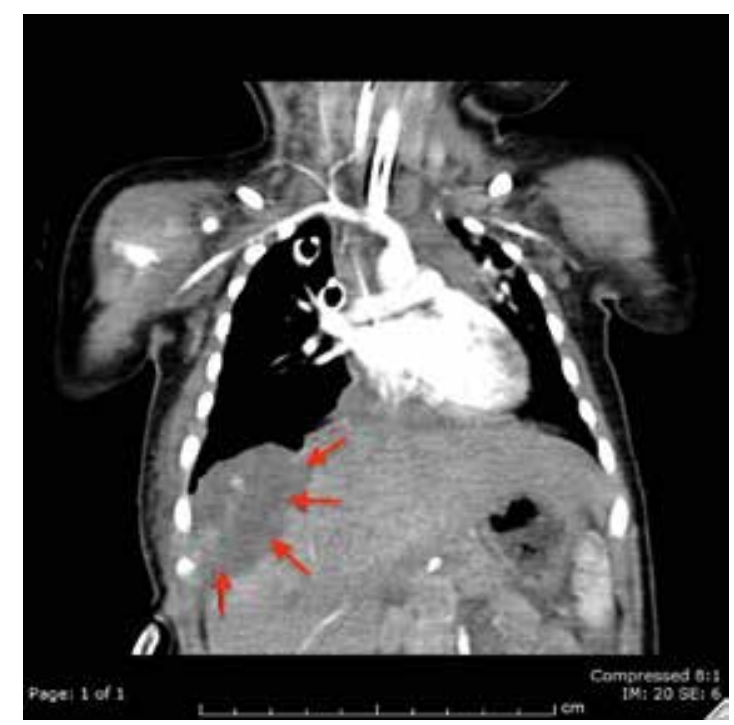

Fig. 3. Contrast-enhanced thorax CT showing $12 \mathrm{~mm}$ diameter nodular-enhanced solid lesions on the right hemithorax inferior pleural face. 

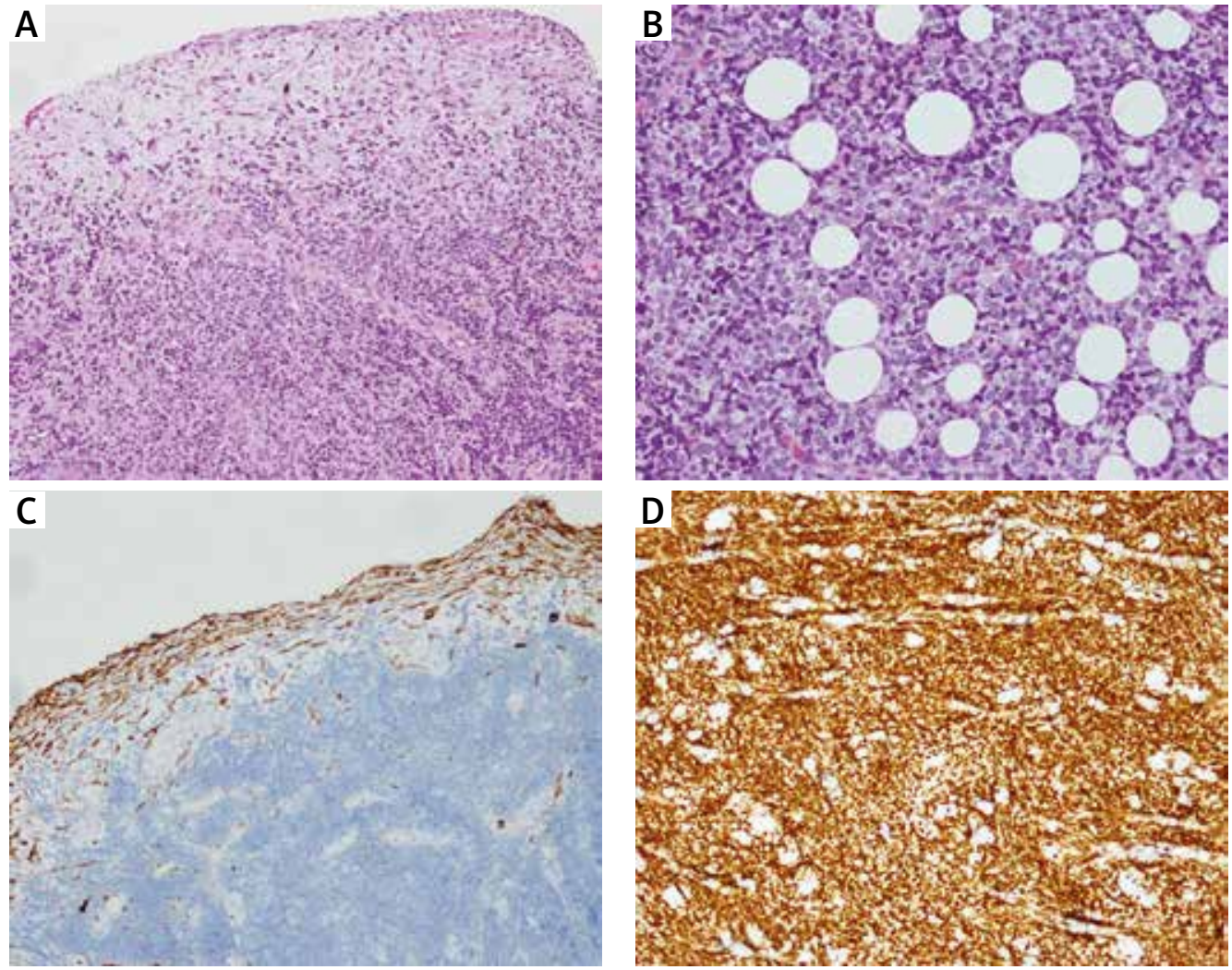

Fig. 4. A) Tumour infiltration consisting of fibroblasts in myxoid stroma in the sub-mesothelial area and small round cells lined with a flattened mesothelium on the surface $(\times 100)$. B) Tumoural cells with clear cytoplasm infiltrating into adipose tissue (HEx200). C) Non-negative staining of positive tumoural cells in pleural plates on the surface with Oscar Keratin dye (Oscar Keratin 100x). D) MYOD1 positivity in tumoral cells $(M Y O D \times 200)$

tumour, and presence of metastasis. Despite the fact that VAC is the gold standard for combination chemotherapy in the treatment of RMS, there are no specific chemotherapy guidelines regarding the treatment for neonates and infants with sarcoma. Infants with RMS are usually treated according to the same protocols used for older children: mainly alkylating agents, vincristine, and actinomycin-D, with or without anthracyclines [12-16]. However, they require ageand weight-adapted doses, given the physiological immaturity of various organs. Pleural effusion regressed, and the thoracic tubes could be taken out just after the first VAC regimen. The primary tumour and the metastasis responded very well after four cycles of chemotherapy with no significant toxicities. Due to possible sequelae, local control, determined by surgery and radiotherapy, also has unique challenges in very young children. In the literature, only three out of 16 patients with congenital ARMS received RT associated with conservative surgery. No conclusion can be drawn concerning the outcome of these small numbers of cases and the lack of follow-up [13]. The Italian Cooperative Group reported a higher local recurrence rate in infants with RMS, who did not receive appropriate local treatment [16]. In our patient, because radiotherapy was not recommended, we decided to continue chemotherapy to 40 weeks. Molecular gene profiling is even more critical in congenital forms of the tumours; despite the fact that the presence of the PAX3/FOXO1 fusion gene shows poor prognosis, other more in-depth biological studies are crucial for congenital RMS patients.

\section{Conclusions}

Our case is a rare alveolar subtype of congenital RMS occurring in the pleural localisation. This is a highly malignant tumour with an abysmal prognosis with almost no long-term survivors. Physicians should be aware of such an unusual presentation of congenital RMS, the diagnosis of which required recurrent pleural tube drainage and internalisation to PICU due to persistent pleural effusion. Although no specific chemotherapy guidelines regarding the treatment for infants with sarcoma exist, good chemotherapy responses are achieved with the regimens used for older children. Molecular biology studies are mandatory for tumour characterisation and the discovery of new therapeutic targets.

The authors declare no conflict of interest.

\section{References}

1. Healy JN, Borg MF. Paediatric nasopharyngeal rhabdomyosarcoma: A case series and literature review. J Med Imaging Radiat Oncol 2010; 54: 388-394

2. Moorthy NL, Prasad BT, Sakunthala P, Bhramaramba K. Alveolar rhabdomyosarcoma of mediating - A case report. Indian J Radiol Imaging 2006; 4: 655-656.

3. Bouchikhi AA, Mellas S, Tazi MF, et al. Embryonic paratesticular rhabdomyosarcoma: a case report. J Med Case Rep 2013; 7: 93.

4. Little DJ, Ballo MT, Zagars GK, Pisters PW, Patel SR, El-Naggar AK, Garden AS, Benjamin RS. Adult rhabdomyosarcoma: outcome following multimodality treatment. Cancer 2002; 95: 377-388. 
5. Nishida Y, Tsukushi S, Urakawa H, Sugiura H, Nakashima H, Yamada Y, Ishiguro N. High incidence of regional and in-transit lymph node metastasis in patients with alveolar rhabdomyosarcoma. Int J Clin Oncol 2014; 19: 536-543.

6. Lawrence W Jr, Anderson JR, Gehan EA, Maurer H. Pretreatment TNM staging of childhood rhabdomyosarcoma: a report of the Intergroup Rhabdomyosarcoma Study Group. Children's Cancer Study Group. Pediatric Oncology Group. Cancer 1997; 80: 11651170.

7. Ferrari A, Casanova M, Bisogno G, et al. Rhabdomyosarcoma in infants younger than one year old: a report from the Italian Cooperative Group. Cancer 2003; 97: 2597-2604.

8. Ragab AH, Heyn R, Tefft M, Hays DN, Newton WA Jr, Beltangady $M$. Infants younger than 1 year of age with rhabdomyosarcoma. Cancer 1986; 58: 2606-2610.

9. Salloum E, Flamant F, Rey A, Caillaud JM, Friedman S, Valteau D, Lemerle J. Rhabdomyosarcoma in infants under one year of age: experience of the Institut Gustave-Roussy. Med Pediatr Oncol 1989; 17: 424-428.

10. Orbach D, Rey A, Oberlin O, Sanchez de Toledo J, Terrier-Lacombe MJ, van Unnik A, Quintana E, Stevens MC. Soft tissue sarcoma or malignant mesenchymal tumors in the first year of life: experience of the International Society of Pediatric Oncology (SIOP) Malignant Mesenchymal Tumor Committee. J Clin Oncol 2005; 23: 4363-4371.

11. Lobe TE, Wiener ES, Hays DM, et al. Neonatal rhabdomyosarcoma: the IRS experience. J Pediatr Surg 1994; 29: 1167-1170.

12. Carli M, Colombatti R, Oberlin O, et al. European intergroup studies (MMT4-89 and MMT4-91) on childhood metastatic rhabdomyosarcoma: final results and analysis of prognostic factors. J Clin Oncol 2004; 22: 4787-4794.

13. Rodriguez-Galindo C, Hill DA, Onyekwere O, et al. Neonatal alveolar rhabdomyosarcoma with skin and brain metastases. Cancer 2001; 92: 1613-1620.

14. Bisogno G, Ferrari A, Prete A, et al. Sequential high-dose chemotherapy for children with metastatic rhabdomyosarcoma. Eur J Cancer 2009; 45: 3035-3041.

15. Sarkar D, Ray S, Saha M, Chakrabarti P. Alveolar rhabdomyosarcoma with multiple distal metastases. A case report and review of literature. BMJ Case Rep 2012; 2012: bcr2012006523. Erratum in: BMJ Case Rep 2012; 2012.

16. Ferrari A, Casanova M, Bisogno G, et al. Rhabdomyosarcoma in infants younger than one year old: a report from the Italian Cooperative Group. Cancer 2003; 97: 2597-2604.

\section{Address for correspondence}

\section{Can Yilmaz Yozgat}

Faculty of Medicine

Bezmialem Vakif University

Adnan Menderes Blv, Vatan Caddesi

34093, Istanbul, Turkey

e-mail: yozgatyilmaz@gmail.com

Submitted: 26.04.2020

Accepted: 1.06 .2020 